\title{
Visual ECG Analysis in Real-world Scenarios
}

\author{
Bernardo Breve, Loredana Caruccio, Stefano Cirillo, Vincenzo Deufemia, Giuseppe Polese \\ Department of Computer Science \\ University of Salerno, Italy \\ \{bbreve,lcaruccio,scirillo,deufemia,gpolese\}@unisa.it
}

\begin{abstract}
Cardiac arrhythmia is an alteration of the heart rhythm, for which the heartbeat is irregular. Based on the severity of this condition, an arrhythmia could represent a serious danger for a patient. An ECG is a graphic representation of an heart rhythm, which provides an overview of heart's conditions over a specific time interval. ECG signal analysis is entrusted to trained clinicians, although complex and frantic environments, such as emergency settings, can make hard to delegate continuous monitoring to the medical personnel. In such scenarios, an automatic detection methodology could provide crucial support in promptly alerting clinicians towards a potential degeneration of a patient's conditions. To this end, we propose a heartbeat classification module capable of capturing the semantics of visual information of ECG signals provided by video frames. The module relies on feature extraction techniques derived from video projected images resulting in ECG data, which are then classified by means of deep-learning models. It can be used to support the early detection of some arrhythmia in critical contexts, such as emergency rooms. We show how the proposed module can be used to support clinicians in this context, and discuss an experimental evaluation performed over ground-truth datasets.
\end{abstract}

Index terms - ECG Analysis, Health scenarios, Arrhythmia, Multimedia

\section{Introduction}

Information and Communication Technology (ICT) as part of the health process is the inclusion of technology in managing and processing information for patients' direct or indirect benefit. In particular, it regards the use of digital devices and software products that allow users to create, store, exchange and use data, with the aim of providing a greater

DOI reference number: 10.18293/DMSVIVA2021-008 degree of efficiency in the management of health information and support professionals both in daily clinical activities in decision-making, such as those designed to identify possible therapies [11]. Particularly challenging are complex and frantic environments, such as emergency settings, where the translation of research evidence into clinical practice is particularly challenging.

Automatic monitoring systems can help professionals in scenarios that require continuous monitoring, and possibly alerting methods to catch the attention of clinicians when specific events occur $[5,8]$. Such systems are becoming even more complex, and try to use different kinds of (smart) devices, which include modules typically focusing on specific tasks. Among these, Machine Learning (ML)-based modules can be used to classify monitored parameters and possibly to provide early detection of some pathologies.

Nevertheless, real-world contexts do not allow human pre-processing phases over raw data to be validated, and automatic support should also be devoted to simplifying the eliciting, processing, and visualizing of data $[6,10]$, trying to provide the best possible quality of data [7]. Thus, complete processes should be managed in real-world scenarios trying to not damage the accuracy that is fundamental in the health domain.

By focusing on the ECG Analysis task, in this paper we considered emergency rooms as basic scenario, where a patient can be monitored through several tools showing his/her vital signs (among which ECG signals can also be included), and several video recording devices are devoted to monitor patient's status and behavior [9]. Clinicians periodically check the patient's status by verifying his/her vital signs, and by interpreting audio/video ECG representations, and identifying the best evidence to guide clinical practice. At the same time, automatic support can be welcomed if it permits to help in solicitous monitoring and recording of clinical parameters and direct clinical inspection.

The proposal is focused on a new module that permits to classify heartbeats towards the early detection of arrhythmia by directly analyzing the visual representation of ECG signals. It aims to provide support in critical clinical sce- 
narios, where the continuous monitoring of patients cannot be accomplished constantly. In fact, by integrating such a module in a more complex monitoring system, it is possible to manage proper alarm triggering components to alert clinicians with the deterioration of ECG parameters related to the presence of some arrhythmia [22].

The module is able to analyze visual representation of ECGs, characterize proper features by considering the correspondent digital representation, and classify, through a ML model, the different types of signals according to the EC57 standard released by the Association for the Advancement of Medical Instrumentation (AAMI) [1]. The module is scalable towards different feature extraction techniques, and different ML models, and is able to provide labels on video frames in accordance with classification results.

The remainder of the paper is organized as follows: Section 2 presents the related work illustrating the main approaches of ECG analysis tasks provided in the literature. In Section 3, we introduce the proposed module by also describing the integrated models and techniques. Experimental results are discussed in Section 4. Finally, Section 5 provides the conclusion and future developments.

\section{Related Work}

Several studies have applied deep learning methodologies in ECG signal analysis. In [13], the authors provided a systematic literature review highlighting the main opportunities of ECG data analysis through shallow and deeplearning techniques, classifying the proposals based on the tasks they could perform:

- Disease detection. This task aims to detect anomalies in a patient's heartbeats, solutions for detecting arrhythmia, myocardial infarction, atrial fibrillation, and congestive heart failure, could represent important support to doctors for speeding up the diagnosis formulation;

- QRS annotation. The application of shallow and deeplearning techniques in the ECG analysis could be significant in annotating QRS waves that identify a single beat. Indeed, for factors depending both on the patient and the medical device, the conformation of the signal could present waves of different morphology, which could sometimes appear difficult to classify in a short amount of time;

- Analysis of sleep patterns. This application scenario could benefit from the application of ECG data analysis. In particular, the presence of sleeping syndromes such as obstructive sleep apnea could be classified through monitoring the ECG signals during sleep;
- Biometric authentication. With the advent of personal wearable devices capable of monitoring heartbeats and, for some recent devices, even provide an ECG analysis, some studies focused on trying to exploit this data as a biometric authentication means to improve the security of smart devices;

- Denoising ECG signals. Machine learning techniques for ECG data also served an important contribution in the steps of denoising signals as a result of an acquisition process.

In [4], authors presented a review of literature on heartbeat abnormality detection. In particular, Annam et al. propose a taxonomy of solutions of the arrhythmia distinguished in two main approaches: feature-based classification and timeseries-based classification. While with timeseries approaches the ECG are entirely and directly processed, in the feature-based classification approach, data are analyzed and decomposed to extract main features, which will then be employed for classifying ECG signals. For each major category, the authors also provided a further splitting between solutions that apply intra-patient or inter-patient categorizations. In the former, data concerning a single patient is partitioned between training and test sets. On the contrary, by applying an inter-patient paradigm, data from different patients are used to assess a strong generalization capability for unknown patient's data.

In [16], Kachuee et al. proposed a solution based on deep convolutional neural network $(\mathrm{CNN})$ for the classification of the ECG heartbeat in different categories. Through a transferable representation, they designed a model for detecting arrhythmia. The authors then proved that this representation appears to be applicable in the prediction of myocardial infarction. The CNN defined obtained results comparable to the state-of-the-art in the classification of ECG signals, while it achieved better results in myocardial infarction classification than most of other proposed solutions.

In [25], the authors proposed a combination of wavelet transform and autoregressive models as a feature extraction method. Then, through the usage of SVM to classify the ECG heartbeat. The Wavelet transform is used for extracting the coefficients of the transform as characteristics of each ECG segment. The Autoregressive Model was also used for extracting coefficients, which combined with the wavelet coefficients, forming a vector of 32 dimensions for classification. The evaluation is then performed through an empirical study based on the MIT-BIH Arrhythmia Database.

In [23], a hierarchical ML method derived from the gradient boosting decision tree has been proposed. It extracts a large number of features from 6 categories (statistics, intervals, etc.) from pre-processed heartbeats via the Wavelet transform. The authors then used recursive feature elimi- 
nation (RFE) to select the most relevant ones. The method has been applied to an inter-patient experiment conforming AAMI standard alongside several contrast experiments.

Instead, the method proposed in [3] by Alarsan et al. is based on Gradient Boosted Trees, Decision Tree and Random Forest, and uses the discrete Wavelet transform for features extraction. It has been evaluated and validated on the MIT-BIH arrhythmia dataset.

Finally, in [26] Zhou et al. propose an architecture composed of ResNet + Bi-LSTM, which combines the benefits of both to extract relevant characteristics and to carry out the classification of ECG signals.

All the works mentioned above are limited to the classification of individual ECG signals extracted from images. Differently to them, we propose a new ML-based module to directly classify in real-time multimedia representations of ECG signals, yielding to the early detection of arrhythmia.

\section{Monitoring ECG Heartbeats}

The early detection of Arrhythmia or other pathologies through ML models is mainly accomplished by directly considering numerical ECG data. Nevertheless, in order to get classification processes applicable in different realworld scenarios, it is necessary to consider different types of raw data including images and/or video sequences. In what follows, we describe the proposed module by introducing different pre-processing steps based on the type of input data (Section 3.1), the CNN architectures we considered to perform some classification tasks (Section 3.2), and the real-world scenarios in which the proposed module can be applied (Section 3.3).

\subsection{Preprocessing}

The imbalance problem is one of the main issues that should be addressed in ML tasks during pre-processing steps. It entails adjusting class imbalance possibly characterizing the prior data distribution over classes. Sampling methods include undersampling andoversampling methods [2], yielding the possibility to remove samples, or add new samples even by creating copies of existing ones.

Figure 1 shows the application of resampling methods on the MIT-BIH dataset [20,21], which represents a sample of the MIT-BIH arrhythmia database, including five classes of ECG signals (N, S, V, F, and U) in accordance with the EC57 AAMI standard [1]. In particular, an undersampling technique has been applied in order to randomly reduce the number of occurrences of signals labeled as " $\mathrm{N}$ ". Moreover, oversampling techniques have been used to randomly increase the samples mapping the other classes of ECG signals (i.e., S, V, F, and U). Thus, the right plot in Figure 1 shows the balanced MIT-BIH dataset after the performed resampling process.

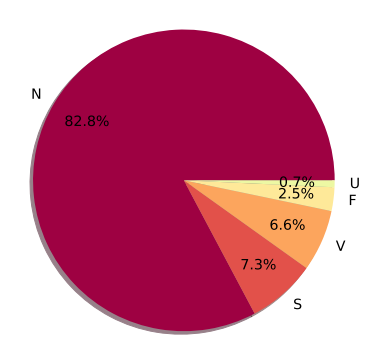

Before resampling.

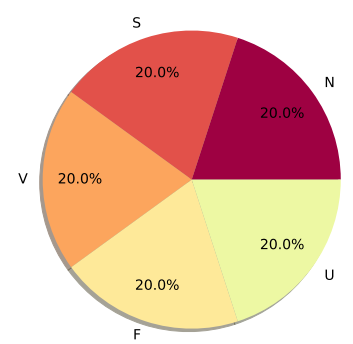

After resampling.
Figure 1: Distribution of classes for MIT-BIH dataset.

When the ECG data to analyze are images or video frames further pre-processing steps should be applied, with the aim to remove potential noise over multimedia data, by cleaning, resizing and recoloring images. To this end, we considered two main techniques based on Red Green Blue (RGB) and Hue Saturation Value (HSV) models, respectively. They allowed us to emphasize the ECG signal with respect to the image background.

The next step concerns the extraction of the feature vector, by firstly detecting peaks over the signal [15], then reconstructing QRS waves around the peaks, and then summarizing and normalizing them in the range $(0,1)$.

\subsection{Convolutional Neural Networks}

CNNs represent one of the most popular classes of artificial neural networks capable of classifying images and/or video sources. This type of neural network has been first proposed in [18] and since then, it has achieved considerable success in several domains, and particularly in the field of health technology [14]. The architecture of a CNN is composed of one or more convolutional layers, and a fully connected layer at the top, containing weights and pooling layers.

In this paper, we adopt three different CNNs to identify arrhythmic diseases from real ECGs, composed of 14, 13, and 31 layers, respectively. Table 1 shows the architecture details of the proposed CNN. In particular, the Input Layer receives an image of dimensions $186 \mathrm{x} 1$, where 186 represents the features obtained after the preprocessing steps on the image. Then, the first block contains a Convolution1D characterized by 64 kernels larger than 6 . It is important to notice that, this value is reduced in the following layers allowing $\mathrm{CNN}$ to further synthesize the images. In fact, due to the several convolutional layers, it is necessary to reduce the size of the kernels in order to avoid losing too much information. Next, a Batch Normalization Layer allows the CNN to normalize and scale the analyzed data. At the end of each 


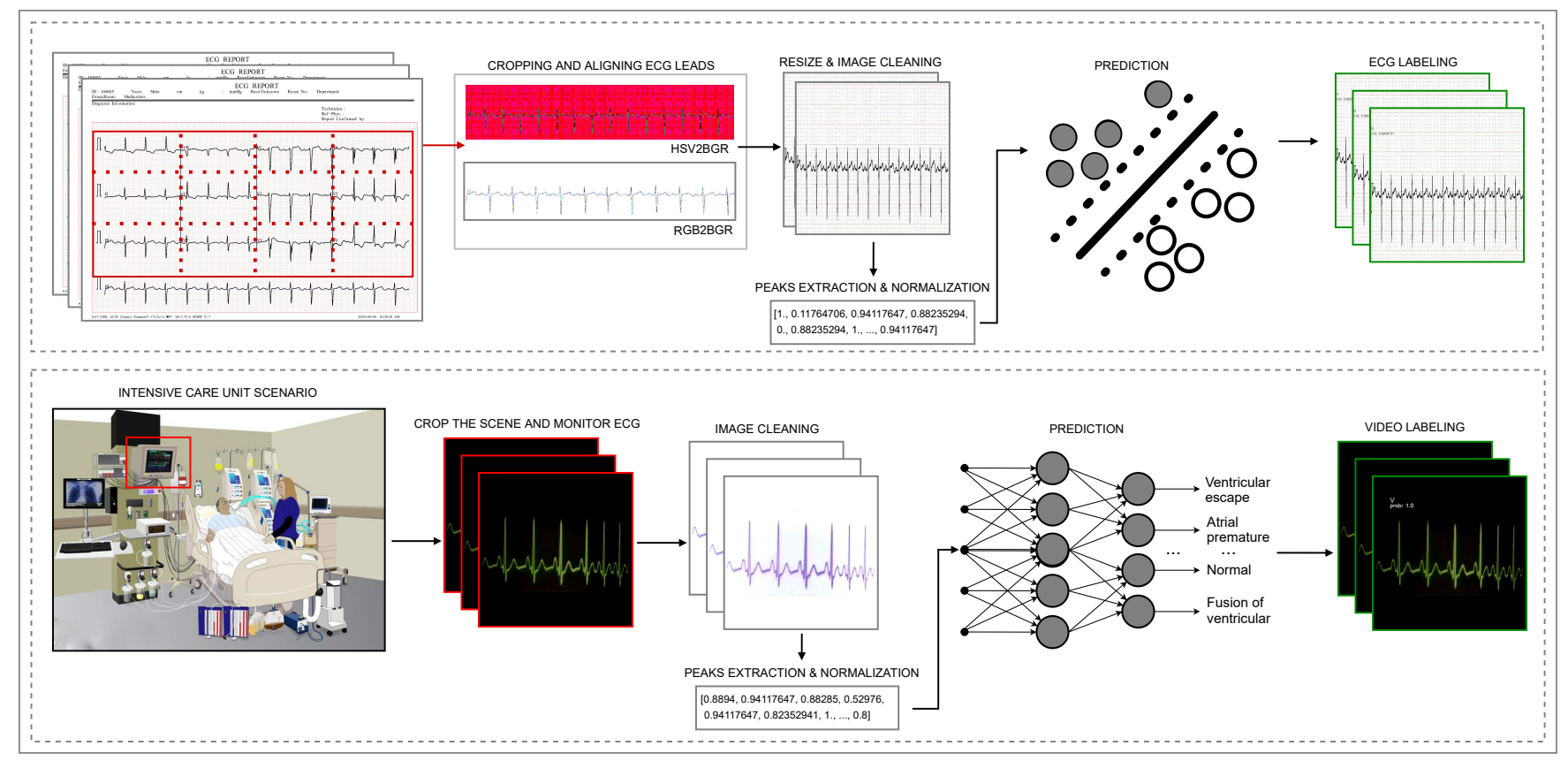

Figure 2: Classification of real ECG report and video frames.

\begin{tabular}{rlll}
\hline Layers & Type & Output Shape & Kernel Size \\
\hline 0 & Input Layer - ECG & $186 \times 1$ & - \\
1 & Convolution1D & $181 \times 64$ & 6 \\
2 & BatchNormalization & $181 \times 64$ & - \\
3 & Maxpooling1D & $91 \times 64$ & 3 \\
4 & Convolution1D & $89 \times 64$ & 3 \\
5 & BatchNormalization & $89 \times 64$ & - \\
6 & Maxpooling1D & $45 \times 64$ & 3 \\
7 & Convolution1D & $43 \times 64$ & 3 \\
8 & BatchNormalization & $43 \times 64$ & - \\
9 & Maxpooling1D & $22 \times 64$ & 3 \\
10 & Flatten & 1408 & - \\
11 & Dense1 & 64 & - \\
12 & Dense2 & 32 & - \\
13 & Output Layer & 5 & - \\
\hline
\end{tabular}

Table 1: Architecture of the first CNN.

block, it has been introduced a Pooling Layer that exploits the Max-Pooling algorithm to perform a down-sampling operation to reduce the feature size.

As we can see, in this first CNN, we consider three similar blocks that differ from the others only in the size of the kernels. The Flatten Layer at the end of these blocks allows $\mathrm{CNN}$ to prepare the data to classify signals with a Neural Network (NN). In fact, two Fully Connected layhave been included in the architecture, with 64 and 32 neurons, respectively. Finally, the Output Layer has been composed of 5 neurons, where 5 represents the number of different arrhythmic diseases to be classified.

\begin{tabular}{rlll}
\hline Layers & Type & Output Shape & Kernel Size \\
\hline 0 & Input Layer - ECG & $186 \times 32$ & - \\
1 & Convolution1D & $185 \times 32$ & 3 \\
2 & Convolution1D & $183 \times 32$ & 3 \\
3 & Maxpooling1D & $91 \times 64$ & 3 \\
4 & Convolution1D & $83 \times 64$ & 5 \\
5 & Convolution1D & $79 \times 64$ & 5 \\
6 & Maxpooling1D & $39 \times 64$ & 3 \\
7 & Convolution1D & $31 \times 128$ & 5 \\
8 & Convolution1D & $27 \times 128$ & 5 \\
9 & Maxpooling1D & $13 \times 128$ & 3 \\
10 & Flatten & 1664 & - \\
11 & Dense & 64 & - \\
12 & Output Layer & 5 & - \\
\hline
\end{tabular}

Table 2: Architecture of the second CNN.

Table 2 details the architecture of the second CNN. Similar to the first model, this has been designed by considering an Input Layer and three similar blocks. However, this CNN considers two different convolutional layers in each block and a Pooling Layer that exploits the algorithm MaxPooling. Unlike the first model, this CNN uses a dilated convolution with $k=2$ that allows increasing the receptive vision (global vision) of the network and linear parameters. In particular, given a 2D image as input, setting the dilation rate $k=1$ represents a normal convolution, $k=2$ means skipping one pixel per input, and $k=4$ means skipping 3 pixels. The resulting data from these layers are processed by a Fully Connected Layer with 64 neurons and an Output 
layer of 5 neurons.

The latest model has been introduced in [16], and represents one of the most recent proposals existing in the literature. In fact, experimental evaluations have shown that this model considerably improves the performance of many existing models.

In the next section, an experimental evaluation of CNNs will be made by applying a cross-validation technique to estimate the skill of each model. The evaluation of these three models allowed us to directly compare the performance of the proposed $\mathrm{CNN}$ with some of the most accurate models in the literature.

\subsection{Applications in real-word scenarios}

To highlight the effectiveness of the proposed module, we identified two real-world scenarios in which it could play a crucial role. In fact, as mentioned before, the frenetic pace at which the emergency rooms work does not always allow the specialized personnel to continuously monitor patients' ECGs. With the adoption of the proposed module, the healthcare personnel could be relieved of this task, being able to be called into question only if some abnormalities are detected by the model.

Figure 2 introduces the process of ECG signal classification in the aforementioned scenarios. They differ on the source from where an ECG signal is taken from. Indeed, data could be acquired both from an ECG report, printed out from an ECG machinery, and directly extrapolated from video frames, which could be recorded by video surveillance cameras.

\subsubsection{Classification of ECG signal from report}

We consider a scenario in which many patients have been performed an ECG, and the personnel has to end-up with many reports to analyze and process. To this end, in order to speed up the analysis process, the reports can be provided sequentially to the proposed module for the analysis.

The structure of an ECG report is composed of 12 leads, each of which represents the status of the heart read from different sensors positioned on the patient's body. For having a proper classification of the ECG, each lead needs to be taken into account, since they provide a different point of view of the heart conditions. Hence, the first step for this analysis is to isolate the 12 different leads that the ECG is composed of. After that, the leads follow the pre-processing, detailing, and cleaning activities detailed in Section 3.1.

The feature vector describing the QRS waves is then normalized and provided to the classifier. The output label of the classification process is added to the ECG report itself through post-processing image editing. The labeled ECG

\begin{tabular}{llll}
\hline Dataset & Data type & \# Samples & Heartbeats classes \\
\hline MIT-BIH & ECG data & 87554 & EC57 AAMI standard \\
PubMed & ECG images & 1937 & Binary \\
\hline
\end{tabular}

Table 3: Statistics on the considered datasets.

\begin{tabular}{lrrr}
\hline \multirow{2}{*}{ Dataset } & Model & \multicolumn{2}{c}{ Times } \\
\cline { 3 - 4 } & Preprocessing (s) & Training (s) \\
\hline \multirow{2}{*}{ MIT-BIH } & Model (1) & & 1873.3 \\
& Model (2) & 0.93 & 1937.6 \\
Model (3) & & 2221.2 \\
PubMed & SVM & 1.2 & 1.4 \\
\hline
\end{tabular}

Table 4: Time performances on the considered datasets.

report is then printed for the personnel to evaluate the response.

\subsubsection{Classification of ECG signal from video frames}

The process of classifying and ECG signal from a video requires a different pre-processing approach. Indeed, in this scenario data are retrieved from video surveillance devices, which most of the time are not directly placed in front of the medical devices in charge of monitoring the patient. To extract ECG data the frame needs to be cropped, isolated, and occasionally transformed in order to flatten the detail for the ECG signal. The obtained single-lead ECG sequences are then processed as done in the previous approach.

Finally, after the prediction of the signal over a single frame, it is labeled and put on hold, waiting for more frames to be processed. After a certain amount of frames is processed, they are reassembled, producing a video sequence identical to the one in input, but with the label classifying the information provided by the signal.

\section{Experimental evaluation}

To evaluate the effectiveness of the proposed module and the considered CNNs, we performed two different experimental sessions. The first is mainly devoted to evaluate the accuracy of CNNs, whereas in the second session, we considered a novel ground-truth dataset, which allowed us to directly consider ECG images as input for both training and validation tasks.

\subsection{Experimental settings}

As mentioned above, we considered 2 different groundtruth datasets: MIT-BIH [20, 21] and PubMed [17], whose statistics are reported in Table 3.

MIT-BIH represents a sample of the MIT-BIH arrhythmia database, including five classes of ECG signals $(\mathrm{N}, \mathrm{S}, \mathrm{V}$, 

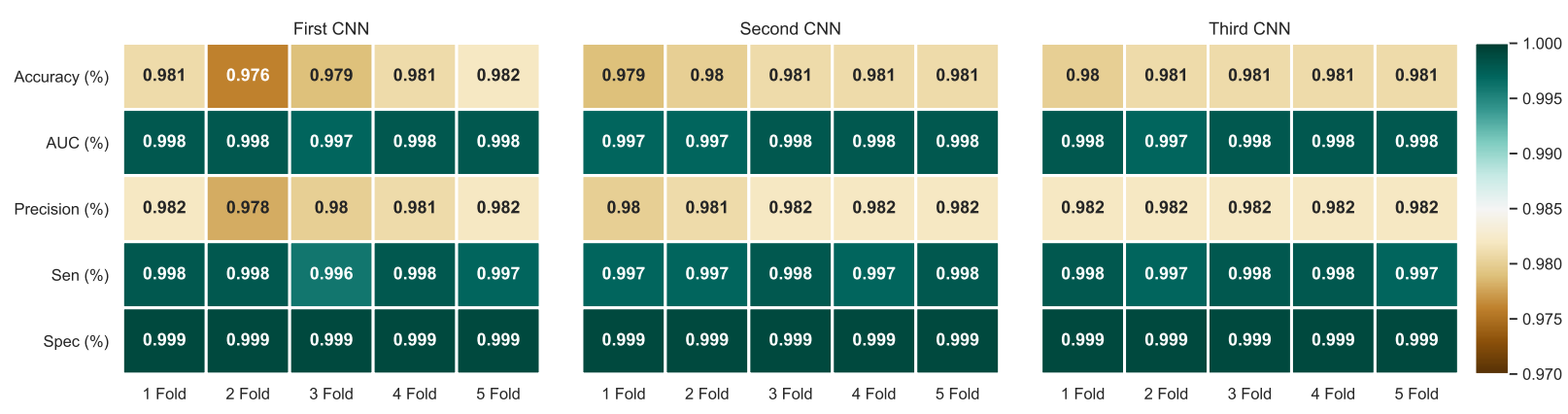

Figure 3: Cross-validation performance for MIT-BIH dataset.

F, and U) in accordance with the EC57 AAMI standard [1]. The first class represents samples with no anomaly detected, $\mathrm{S}$ and $\mathrm{V}$ classes categorize different types of heartbeat anomalies, class $\mathrm{F}$ includes ECGs with combined features from $\mathrm{S}$ and $\mathrm{V}$ classes, whereas $\mathrm{U}$ represents the class of unrecognized heartbeats. The MIT-BIH Arrhythmia Database is one of the most used datasets for evaluating automatic ECG Analysis techniques, and it contains ECG samples obtained from 47 subjects, and recorded in a 30 min duration and sampled at $360 \mathrm{~Hz}$. We used such dataset for evaluating the effectiveness of the two considered CNNs.

PubMed represents a novel dataset containing ECG images of Cardiac and COVID-19 patients collected in Cardiac Care and Isolation Units of different health care institutes across Pakistan. Image have been classified in five distinct categories (COVID-19, Abnormal Heartbeat, Myocardial Infarction (MI), Previous History of MI, and Normal Person). In our evaluation, we considered a sample of 1937 images including Abnormal Heartbeat and Normal Person images, yielding a binary classification task. We used such dataset for evaluating the effectiveness of the whole proposed methodology.

The time performances for the preprocessing steps are shown in Table 4. As we can see, the average preprocessing time for each image is less than 1 second for the MIT-BIH dataset, and 1.2 seconds for the PubMed dataset. In the latter case, the preprocessing step takes longer due to the identification of each lead, as described in Section 3.3.

Moreover, the performance of the classification have been evaluated in each task by using several evaluation metrics. In particular, let TP, TN, FP, and FN be the values of True Positive, True Negative, False Positive, and False Negative, respectively, then we can define the evaluation metrics as follow:

- Area Under the Curve (AUC) represents the classifier's ability to distinguish classes;

- Precision (Prec) represents the number of positives that are correct, over all identified positives: Prec $=\frac{\mathrm{TP}}{\mathrm{TP}+\mathrm{FP}}$
- Sensitivity (Sen), also named Recall (Rec), represents the proportion of positives that are correctly identified: $\mathrm{Sen}=\frac{\mathrm{TP}}{\mathrm{TP}+\mathrm{FN}}$

- Specificity (Spec) represents the proportion of negatives that are correctly identified: $\mathrm{Spec}=\frac{\mathrm{TN}}{\mathrm{TN}+\mathrm{FP}}$

- Accuracy (Acc) represents the number of positives and negatives correctly identified over the over the total number of tested elements: $\mathrm{Acc}=\frac{\mathrm{TP}+\mathrm{TN}}{\mathrm{TP}+\mathrm{TN}+\mathrm{FP}+\mathrm{FN}}$

- F1-score (F1) represents the armonic mean of precision and recall: $\mathrm{F} 1=2 \times \frac{\text { Prec } \times \text { Rec }}{\text { Prec }+ \text { Rec }}$

\subsection{Evaluating $\mathrm{CNN}$ performances}

To evaluate the accuracy and the effectiveness of predictive models, it is necessary to perform one or more evaluations of the errors obtained from the models. Generally, after the training phase, the model error is calculated in order to provide a numerical estimate considering the difference between the expected and original responses, called training error. However, this type of evaluation only provides a general idea of how the model works on the considered data. To this end, it is necessary to adopt cross-validation techniques that allow to accurately evaluate the performance of the models. These techniques do not use the entire dataset for the training phase. In fact, only a portion of data are used for training the model. The rest of data are used to evaluate the performance of the model.

Among the most reliable cross-validation techniques, Kfold [24] is one of the most popular which provides for randomly dividing data into $\mathrm{k}$ groups of approximately equal size, called folds. The first fold is kept for testing, while the model is trained on the $k-1$ folds. Thus, this validation process is repeated $k$ times considering different folds each time. Figure 3 shows the results after the K-fold validation of each considered CNN model over the $80 \%$ of data for training step, and the remaining $20 \%$ for testing with 5 different folds (i.e., $k=5$ ). 


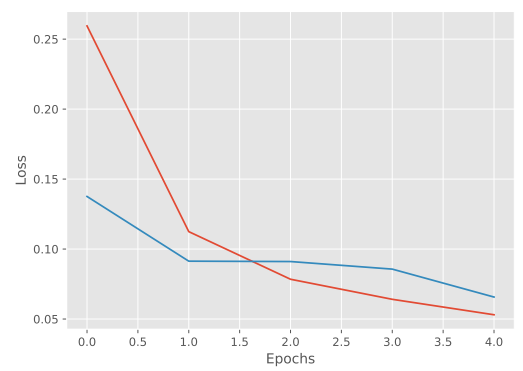

(a) Loss of the model (1).

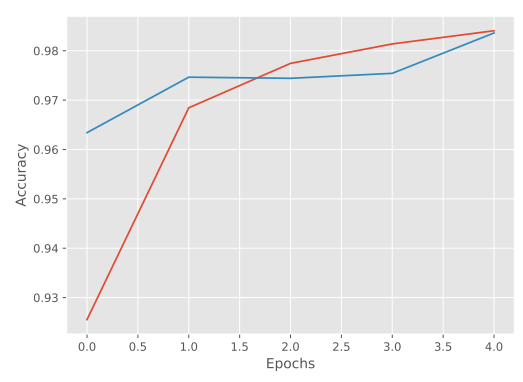

(d) Accuracy of the model (1).

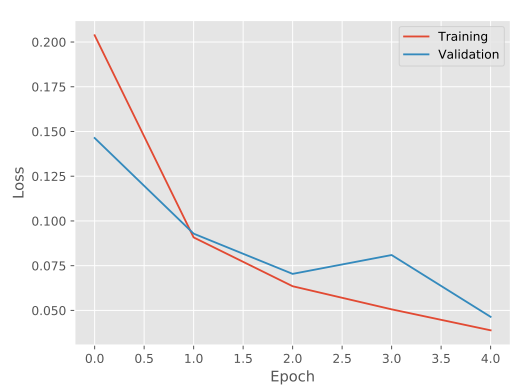

(b) Loss of the model (2).

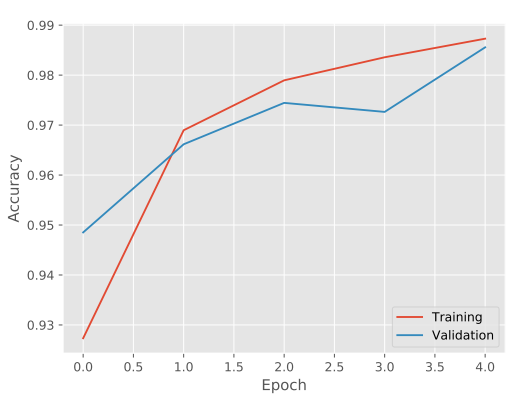

(e) Accuracy of the model (2).

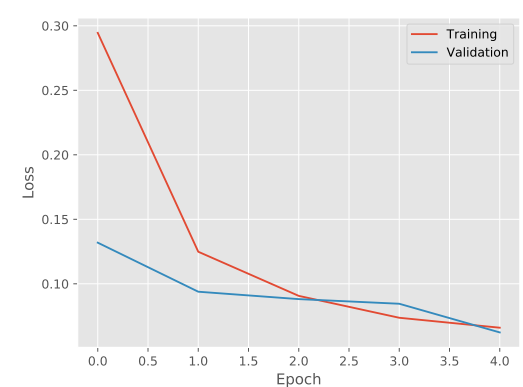

(c) Loss of the model (3).

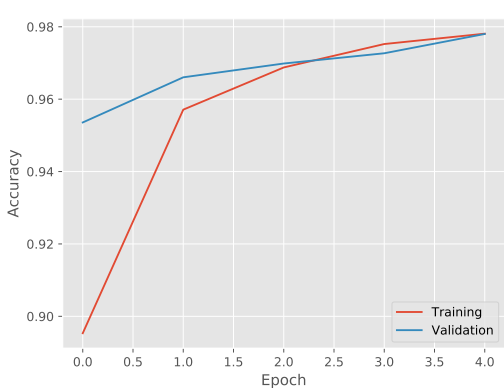

(f) Accuracy of the model (3).

Figure 4: Learning curves of the considered CNN models.

Results over aforementioned metrics are detailed in Figure 3, which shows an accuracy value that reaches its maximum in the last fold for the first CNN (98.2\%), whereas this value stabilizes already from the first fold for the other CNNs, which have an accuracy value of $98.1 \%$. Moreover, the overall average values of the considered CNNs are $99.7 \%, 99.9 \%, 99.7 \%$, and $98.2 \%$ for Sen, Spec, AUC, and Precision, respectively.

The learning curves, namely loss and accuracy, for all considered CNN models are shown in Figure 4. In general, learning curves plot performances on the training validation sets as a function of the training epochs, in terms of loss or accuracy. They can also identify under/over-fitting problems. By comparing plots of the considered models, they show very similar trend for the training line, whereas little differences in the trend monotonicity are shown for validation line. Nevertheless, none of the considered models seem to present the aforementioned problems.

Finally, classification results in terms of precision, recall, and F1-measure obtained over single classes or in average, are shown in Figure 5. We can notice that the second model obtains discordant results across different classes; whereas the other two models present less variability. In general, the fist model can be considered the best performing one.

\subsection{Classification of ECG images}

In the following section, we will discuss how we evaluated the proposed module according to the scenario presented in Section 3.3.1.

In details, we evaluated the module on the PubMed dataset described above. Starting from a total of 51712 leads ECG reports divided between 284 ECGs showing normal heart activity, and 233 showing abnormal heartbeat conditions, we applied some pre-processing steps in order to extrapolate information about each ECG. In particular, we first split the ECG isolating the 12-leads it was composed of, and for each lead we extracted peaks with a vector of 186 numerical values. The leads have then been chained forming an array of 2232 integers. After labeling each array according to the type of ECG they were describing, we trained a Support Vector Machine (SVM) classifier, whose application in these scenarios has already been experimented [12].

Figure 6 reports the classification results obtained over a 5 -fold cross validation process. The two datasets, representing normal and abnormal heartbeats, have been randomly shuffled in training and testing datasets, respectively, containing the $80 \%$ and $20 \%$ of all available reports. By repeating the same evaluation 5 times varying, as said, in a random way the ECGs applied in the training and testing phases.

This evaluation proved that the preprocessing approach 


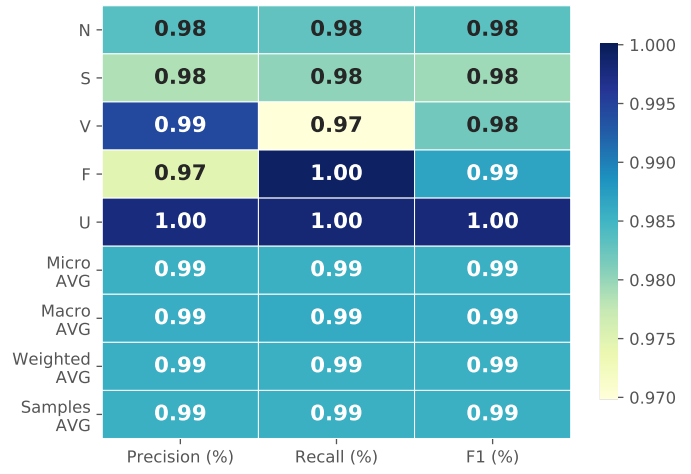

(a) Classification metrics for the first model.

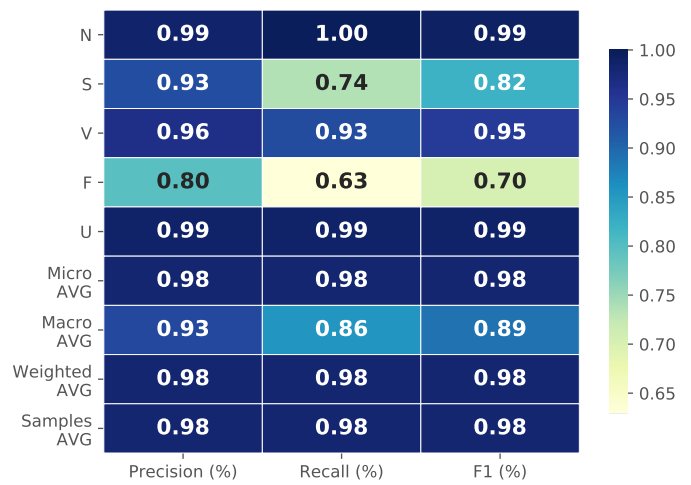

(b) Classification metrics for the second model.

\begin{tabular}{|c|c|c|c|}
\hline $\mathrm{N}-$ & 0.98 & 0.98 & 0.98 \\
\hline s. & 0.98 & 0.97 & 0.98 \\
\hline$v-$ & 0.97 & 0.99 & 0.98 \\
\hline $\mathrm{F}$ - & 0.99 & 0.98 & 0.98 \\
\hline u & 0.99 & 1.00 & 1.00 \\
\hline $\begin{array}{l}\text { Micro } \\
\text { AVG }\end{array}$ & 0.98 & 0.98 & 0.98 \\
\hline $\begin{array}{l}\text { Macro } \\
\text { AVG }\end{array}$ & 0.98 & 0.98 & 0.98 \\
\hline $\begin{array}{l}\text { Weighted } \\
\text { AVG }\end{array}$ & 0.98 & 0.98 & 0.98 \\
\hline $\begin{array}{l}\text { Samples } \\
\text { AVG }\end{array}$ & 0.98 & 0.98 & 0.98 \\
\hline & recision (\%) & Recall (\%) & F1 (\%) \\
\hline
\end{tabular}

(c) Classification metrics for the third model.

Figure 5: Classification metrics of CNN models on MITBIH dataset.

has obtained an average accuracy value of $90 \%$. The average values for AUC and Precision is $92.4 \%$ and $82.6 \%$, respectively. Finally, the average F1-measure value emerged during the evaluation has been 0.92 .

\section{Conclusions}

The automatic detection of arrhythmia from visual ECG data represents a particularly challenging task. Nevertheless, real-world scenarios require some support especially

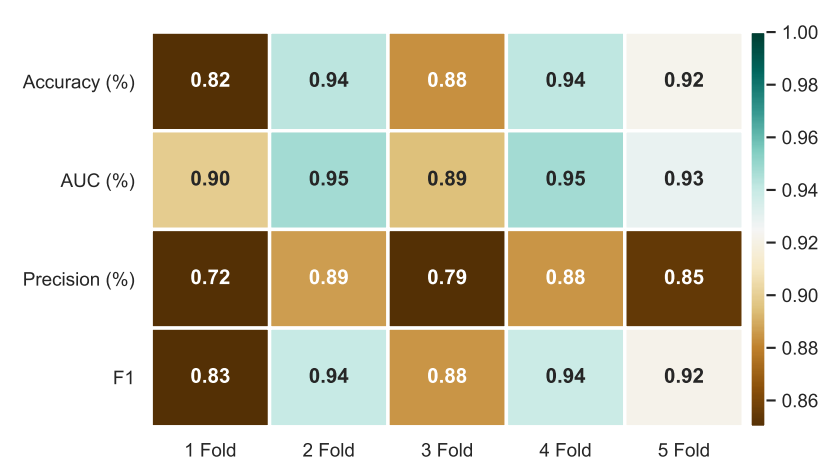

Figure 6: Cross-validation results for PubMed dataset.

to empower the continuous monitoring of patients in frantic settings, such as emergency rooms. The proposed classification module tries to provide some findings in this respect. Although these results can be improved in the future, to the best of our knowledge, they represent the first-ever proposal applying these techniques to real-world scenarios. Modules proposed in literature perform their evaluation on numerical datasets, whereas data is collected and presented directly in their vector representation. Having to deal with images or video frames requires applying more accurate techniques to extract features since issues such as image noise or image distortion are quite common in this scenario. Considering that, the obtained results provide a good starting point for applying these procedures in real-world scenarios.

Future directions should consider such challenging scenarios, by providing further real-world datasets for heartbeat-related pathologies, by also considering data from several IoT sensors [19]. This could entail the analysis of the applicability of such kind of support in real-world settings. Further studies should also be considered in less expressive or distorted ECG visual representations.

\section{References}

[1] AAMI. AAMI EC57- Testing and reporting performance results of cardiac rhythm and st segment measurement algorithms, 2012.

[2] S. M. Abd Elrahman and A. Abraham. A review of class imbalance problem. Journal of Network and Innovative Computing, 1(2013):332-340, 2013.

[3] F. I. Alarsan and M. Younes. Analysis and classification of heart diseases using heartbeat features and machine learning algorithms. Journal of Big Data, 6(1):1-15, 2019.

[4] J. R. Annam, S. Kalyanapu, S. Ch, J. Somala, and S. B. Raju. Classification of ECG heartbeat arrhythmia: A review. Procedia Computer Science, 171:679-688, 2020.

[5] M. Blount, M. R. Ebling, J. M. Eklund, A. G. James, C. McGregor, N. Percival, K. Smith, and D. Sow. Real-time analysis for intensive care: development and deployment of the 
artemis analytic system. IEEE Engineering in Medicine and Biology Magazine, 29(2):110-118, 2010.

[6] B. Breve, L. Caruccio, S. Cirillo, V. Deufemia, and G. Polese. Visualizing dependencies during incremental discovery processes. In EDBT/ICDT Workshops, 2020.

[7] L. Caruccio and S. Cirillo. Incremental discovery of imprecise functional dependencies. Journal of Data and Information Quality (JDIQ), 12(4):1-25, 2020.

[8] L. Caruccio, O. Piazza, G. Polese, and G. Tortora. Secure iot analytics for fast deterioration detection in emergency rooms. IEEE Access, 8:215343-215354, 2020.

[9] L. Caruccio, G. Polese, G. Tortora, and D. Iannone. Edcar: A knowledge representation framework to enhance automatic video surveillance. Expert Systems with Applications, 131:190-207, 2019.

[10] G. Costagliola, V. Fuccella, M. Giordano, and G. Polese. Monitoring online tests through data visualization. IEEE Transactions on Knowledge and Data Engineering, 21(6):773-784, 2008.

[11] G. Fitzpatrick and G. Ellingsen. A review of 25 years of CSCW research in healthcare: contributions, challenges and future agendas. Computer Supported Cooperative Work (CSCW), 22(4-6):609-665, 2013.

[12] C. L. Herry, M. Frasch, A. J. Seely, and H.-t. Wu. Heart beat classification from single-lead ecg using the synchrosqueezing transform. Physiological measurement, 38(2):171, 2017.

[13] S. Hong, Y. Zhou, J. Shang, C. Xiao, and J. Sun. Opportunities and challenges of deep learning methods for electrocardiogram data: A systematic review. Computers in Biology and Medicine, page 103801, 2020.

[14] W. N. Ismail, M. M. Hassan, H. A. Alsalamah, and G. Fortino. CNN-based health model for regular health factors analysis in internet-of-medical things environment. IEEE Access, 8:52541-52549, 2020.

[15] E. Jones, T. Oliphant, P. Peterson, et al. Scipy: Open source scientific tools for python. 2001.

[16] M. Kachuee, S. Fazeli, and M. Sarrafzadeh. ECG heartbeat classification: A deep transferable representation. In 2018 IEEE International Conference on Healthcare Informatics (ICHI), pages 443-444. IEEE, 2018.
[17] A. H. Khan, M. Hussain, and M. K. Malik. ECG images dataset of cardiac and COVID-19 patients. Data in Brief, 34:106762, 2021.

[18] Y. LeCun, B. Boser, J. S. Denker, D. Henderson, R. E. Howard, W. Hubbard, and L. D. Jackel. Backpropagation applied to handwritten zip code recognition. Neural computation, 1(4):541-551, 1989.

[19] P. Lo Giudice, A. Nocera, D. Ursino, and L. Virgili. Building topic-driven virtual iots in a multiple iots scenario. Sensors, 19(13):2956, 2019.

[20] R. Mark, P. Schluter, G. Moody, P. Devlin, and D. Chernoff. An annotated ECG database for evaluating arrhythmia detectors. In IEEE Transactions on Biomedical Engineering, volume 29, pages 600-600, 1982.

[21] G. B. Moody and R. G. Mark. The mit-bih arrhythmia database on cd-rom and software for use with it. In [1990] Proceedings Computers in Cardiology, pages 185188. IEEE, 1990.

[22] E. Özcan, D. Birdja, L. Simonse, and A. Struijs. Alarm in the ICU! Envisioning patient monitoring and alarm management in future intensive care units. Service Design and Service Thinking in Healthcare and Hospital Management, pages 421-446, 2019.

[23] H. Shi, H. Wang, Y. Huang, L. Zhao, C. Qin, and C. Liu. A hierarchical method based on weighted extreme gradient boosting in ECG heartbeat classification. Computer methods and programs in biomedicine, 171:1-10, 2019.

[24] T.-T. Wong and P.-Y. Yeh. Reliable accuracy estimates from $\mathrm{k}$-fold cross validation. IEEE Transactions on Knowledge and Data Engineering, 32(8):1586-1594, 2019.

[25] Q. Zhao and L. Zhang. ECG feature extraction and classification using wavelet transform and support vector machines. In 2005 International Conference on Neural Networks and Brain, volume 2, pages 1089-1092. IEEE, 2005.

[26] Y. Zhou, H. Zhang, Y. Li, and G. Ning. ECG heartbeat classification based on ResNet and bi-LSTM. In IOP Conference Series: Earth and Environmental Science, volume 428, page 012014. IOP Publishing, 2020. 